\title{
De novo assembly and characterization of Camelina sativa transcriptome by paired-end sequencing
}

\author{
Chao Liang ${ }^{1}$, Xuan Liư ${ }^{2}$ Siu-Ming Yiu ${ }^{2}$ and Boon Leong Lim*
}

\begin{abstract}
Background: Biofuels extracted from the seeds of Camelina sativa have recently been used successfully as environmentally friendly jet-fuel to reduce greenhouse gas emissions. Camelina sativa is genetically very close to Arabidopsis thaliana, and both are members of the Brassicaceae. Although public databases are currently available for some members of the Brassicaceae, such as A. thaliana, A. lyrata, Brassica napus, B. juncea and B. rapa, there are no public Expressed Sequence Tags (EST) or genomic data for Camelina sativa. In this study, a high-throughput, large-scale RNA sequencing (RNA-seq) of the Camelina sativa transcriptome was carried out to generate a database that will be useful for further functional analyses.

Results: Approximately 27 million clean "reads" filtered from raw reads by removal of adaptors, ambiguous reads and low-quality reads (2.42 gigabase pairs) were generated by Illumina paired-end RNA-seq technology. All of these clean reads were assembled de novo into 83,493 unigenes and 103,196 transcripts using SOAPdenovo and Trinity, respectively. The average length of the transcripts generated by Trinity was $697 \mathrm{bp}$ ( $N 50=976$ ), which was longer than the average length of unigenes ( $319 \mathrm{bp}, \mathrm{N} 50=346 \mathrm{bp}$ ). Nonetheless, the assembly generated by SOAPdenovo produced similar number of non-redundant hits $(22,435)$ with that of Trinity $(22,433)$ in BLASTN searches of the Arabidopsis thaliana CDS sequence database (TAIR). Four public databases, the Kyoto Encyclopedia of Genes and Genomes (KEGG), Swiss-prot, NCBI non-redundant protein (NR), and the Cluster of Orthologous Groups (COG), were used for unigene annotation; 67,791 of 83,493 unigenes (81.2\%) were finally annotated with gene descriptions or conserved protein domains that were mapped to 25,329 non-redundant protein sequences. We mapped 27,042 of 83,493 unigenes (32.4\%) to 119 KEGG metabolic pathways.

Conclusions: This is the first report of a transcriptome database for Camelina sativa, an environmentally important member of the Brassicaceae. We showed that C. savita is closely related to Arabidopsis spp. and more distantly related to Brassica spp. Although the majority of annotated genes had high sequence identity to those of $A$. thaliana, a substantial proportion of disease-resistance genes (NBS-encoding LRR genes) were instead more closely similar to the genes of other Brassicaceae; these genes included BrCN, BrCNL, BrNL, BrTN, BrTNL in B. rapa. As plant genomes are under long-term selection pressure from environmental stressors, conservation of these disease-resistance genes in C. sativa and B. rapa genomes implies that they are exposed to the threats from closely-related pathogens in their natural habitats.
\end{abstract}

Keywords: Brassicaceae, Camelina sativa, Transcriptome, de novo, Paired-end sequencing, NBS-LRR

\footnotetext{
* Correspondence: bllim@hku.hk

${ }^{1}$ School of Biological Sciences, the University of Hong Kong, Pokfulam, Hong Kong, China

Full list of author information is available at the end of the article
}

\section{Biomed Central}

(c) 2013 Liang et al.; licensee BioMed Central Ltd. This is an Open Access article distributed under the terms of the Creative Commons Attribution License (http://creativecommons.org/licenses/by/2.0), which permits unrestricted use, distribution, and reproduction in any medium, provided the original work is properly cited. 


\section{Background}

Camelina sativa is a dicotyledonous plant in the Family Brassicaceae. It is commonly known as Camelina, "goldof-pleasure" or false flax. It has a growth cycle similar to that of Arabidopsis [1]. Camelina has been cultivated as a source of vegetable oil in Europe, central Asia and North America. The life cycle of $C$. sativa is relatively short, spanning approximately 100-120 days; thus, the species is very suitable for renewable-resource generation and as a spring or fall rotation crop [2]. C. sativa has 3,500 years of cultivation history. Although it is an ancient crop, Camelina cultivation has decreased gradually in modern times in relation to rapeseed [3]. The majority (80\%) of fatty acids in Camelina oil are unsaturated; these are an important source of omega-3 fatty acids [4]. In Camelina seeds, polyunsaturated fatty acids constitute more than $50 \%$ of the total, and linolenic acid (18:3n-3) makes up about $35-40 \%$ of total fatty acids [3]. Camelina is recommended as a dietary supplement because of these benefits. In addition to its dietary use, Camelina oil has non-food applications, such as soaps, varnishes and biodiesel $[5,6]$. Production of this oil may solve the problem of limited feedstock availability for bio-diesel production. Camelina sativa would be useful as an alternative crop for biodiesel due to its low cost of production and high energy content. Because of the relatively high ester yield, alkyl esters from Camelina oil have been used as biodiesel [7]. Camelina oil has also been used directly as a fuel for diesel transport engines [6]. A further advantage of Camelina seed oil is that it produces less $\mathrm{CO}_{2}$ than traditional mineral oil products [8]. Moreover, Camelina is more drought-resistant and frost-tolerant than rapeseed and can thus be grown on land with little fertilizer or on land that is fallow. In comparison with other oilseed plants, Camelina is particularly competitive on highly saline soils [9]. The species is also well-known for its elevated resistance to insect pests and pathogens [10].

A number of plant transcriptomes have been deeply sequenced and subjected to further analysis over the last decade, particularly in model species of monocotyledons (Oryza sativa) and dicotyledons (Arabidopsis). These analyses provide valuable databases for nonmodel plant species $[11,12]$. However, there is no EST or genomic sequence currently available for Camelina sativa in the GenBank database. Transcriptomic sequence data for this low-cost oilseed plant will provide a valuable source of genomic information for practitioners of plant sciences. In the present study, we adopted Illumina paired-end sequencing to analyze the leaf transcriptome of Camelina sativa. In total, 2.42 gigabase pairs were obtained by deep sequencing; unigenes involved in most metabolic pathways were detected by our procedures. This is the first report of a transcriptome database for this oilseed plant. We provide a public dataset for genetic analysis and biological study of Camelina sativa.

\section{Results}

Sequencing and transcriptome assembly by SOAPdenovo Total RNA was extracted from young and mature leaves of Camelina sativa. Poly $\mathrm{A}^{+}$mRNA was obtained by passing total RNA through a column of beads conjugated with oligo (dT); the product was then fragmented into short sequences (200-700nt). The shortened mRNA was transcribed to cDNA by reverse transcriptase before sequencing. Clean "reads" were filtered from raw reads by removal of adaptors, ambiguous reads and low-quality reads. Approximate 27 million clean reads (2.42 Gbp) with a mean length of $90 \mathrm{bp}$ were obtained (GenBank accession number: SRA057100). Two assembly methods were adopted and compared (Table 1). We used SOAPdenovo to assemble all high-quality clean reads into contigs (37.24 Mbp longer than $75 \mathrm{bp}$ ), which were assembled into scaffolds (32.72 Mbp longer than $100 \mathrm{bp}$ ) that were in turn assembled de novo into unigenes (26.65 Mbp longer than $100 \mathrm{bp}$ ). We generated 204,190 contigs (length $\geq 75 \mathrm{bp}$ ) with a mean length of 182 bp and an N50 of 194 bp (Table 2). The majority of contigs $(175,262)$ were in the range $100-400 \mathrm{bp}$, which accounted for $71.9 \%$ of total contigs $(227,194$ total reads) (Additional file 1: Figure S1a). In order to assemble scaffolds, the contigs were connected with $\mathrm{N}$ to represent unknown sequences between two contiguous contigs. Details of scaffold total number, mean length and the N50 value are given in Table 2. Most of the scaffolds $(118,708 / 129,539=89.5 \%)$ reads had gap length ratios of $<0.01$ (Additional file 1: Figure S1b) and the most frequent lengths (91.6\%) were between 100 and $500 \mathrm{nt}$ (Additional file 1: Figure S1c). After assembling the scaffolds de novo with SOAPdenovo software, we filled scaffold gaps with the lowest number of Ns so that each scaffold could not be extended from either side. Resulting sequences were defined as unigenes. In total,

Table 1 Comparison of SOAPdenovo and Trinity assembly results

\begin{tabular}{lll}
\hline & SOAPdenovo & Trinity \\
\hline Total length (nt) & $26,651,285$ & $71,935,591$ \\
Total number (n) & 83,493 & 103,196 \\
N50 & 346 & 976 \\
Mean length (nt) & 319 & 697 \\
$100-500 \mathrm{nt}$ & 72,616 & 53,377 \\
$500-1000 \mathrm{nt}$ & 9,006 & 27,983 \\
$1000-1500 \mathrm{nt}$ & 1,339 & 12,228 \\
$1500-2000 \mathrm{nt}$ & 354 & 5,623 \\
$\geq 2000 \mathrm{nt}$ & 178 & 3,985 \\
\hline
\end{tabular}


Table 2 Summary details of sequences produced by SOAPdenovo assembly after Illumina sequencing

\begin{tabular}{lllll}
\hline & $\begin{array}{l}\text { Sequences } \\
(\mathbf{n})\end{array}$ & $\begin{array}{l}\text { Base pairs } \\
\text { (Mbp) }\end{array}$ & $\begin{array}{l}\text { Mean length } \\
\text { (bp) }\end{array}$ & N50 (bp) \\
\hline Clean reads & $26,942,130$ & $2,424.79$ & 90 & - \\
$\begin{array}{l}\text { Contigs } \\
(\geq 75 \text { bp) }\end{array}$ & 204,190 & 37.24 & 182 & 194 \\
$\begin{array}{l}\text { Scaffolds } \\
(\geq 100 \text { bp) }\end{array}$ & 129,539 & 32.72 & 253 & 284 \\
$\begin{array}{l}\text { Total } \\
\text { unigenes } \\
(\geq 100 \text { bp) }\end{array}$ & 83,493 & 26.65 & 319 & 346 \\
\hline
\end{tabular}

83,493 unigenes were generated with a mean unigene length of 319 bp and an N50 of 346 bp (Table 2) (GenBank accession number: GABO00000000). There were 10,877 unigenes with $\geq 500 \mathrm{bp}$, and 1,871 unigenes with $\geq 1000 \mathrm{bp}$; the majority of unigenes $(87.0 \%)$ had 100 to $500 \mathrm{bp}$ (Figure S1d). The frequency distributions of unigene lengths and ratios of gap length to size of assembled unigene are depicted in Figures S1d and Figure S1e, respectively. Among all unigenes, 99.87\% (83,386 unigenes) had gap lengths that were $<5 \%$ of total length (Figure S1e). The random distribution of reads in assembled unigenes is presented in Figure S1f to display sequencing bias.

\section{De novo transcriptome assembly by Trinity}

To verify SOAPdenovo assembly results, we also made use of the Trinity assembly method, which assembles full-length transcripts without reference genomes [13]. In total 103,196 transcripts with a total length of 71,935,591 bp were generated (GenBank accession number: GABL00000000), significantly exceeding the output from SOAPdenovo $(26,651,285$ bp for 83,493 unigenes). The mean length and N50 obtained by Trinity assembly were also longer than those obtained by SOAPdenovo (Table 1).
To further verify the assembly results from both methods, we mapped both unigenes (SOAPdenovo) and transcripts (Trinity) to Arabidopsis thaliana (TAIR10_cds_20101214) CDS sequences using BLAT (Table 3). Parameters of BLAT were set as default ( $\operatorname{minMatch}=2$, minScore=30, minIdentity=90, $\operatorname{maxGap}=2$, tileSize $=11$ and stepSize=11). Of 83,493 unigenes, 52,864 were successfully mapped to CDS in TAIR; 70,753 of 103,196 transcripts were also successfully mapped. Of the total CDS number in TAIR, 63.4\% $(22,435 / 35,386)$ and $61.4 \%(22,433 / 35,386)$ were mapped with sequences assembled by SOAPdenovo and Trinity, respectively. Among the mapped CDS in TAIR, 59.6\% $(21,084 / 35,386)$ were generated by both methods. Thus, similar mapping results were obtained from the two assembly methods when the Arabidopsis thaliana genome was taken as a reference genome. We adopted SOAPdenovo output for further analyses.

\section{Annotation of predicted proteins in NR and Swiss-Prot databases}

In total, 67,791 unigenes were annotated with 25,329 unique protein IDs. Most protein IDs (25,075 of 25,329) were annotated by the NR database. To annotate gene names and protein coding sequences among unigenes, we searched all six-frame translations of unigenes against the NR plant protein database in NCBI by running BLASTX with an E-value cut-off 1.0E-5. In total, 67,497 BLASTX hits (matches) were obtained, covering $80.8 \%$ of all unigenes (Table 4 and Additional file 2). Of these, 45,307 and 16,969 BLASTX hits were matched to Arabidopsis thaliana (TAIR 9) and A. lyrata proteins, respectively. Only 722 BLASTX hits were aligned to NR protein of Brassica spp. (Table 4). Thus, Camelina sativa was genetically more closely related to Arabidopsis than Brassica. The E-value frequency distribution of significant hits (E-value $\leq 1.0 \mathrm{E}-50)$ showed that $26 \%$ of the sequences shared strong homologies; the majority of matched sequences (74\%) had E-values in the range

Table 3 Assembly results with TAIR 10.0 CDS using SOAPdenovo and Trinity software

\begin{tabular}{lll}
\hline & SOAPdenovo & Trinity \\
\hline Number of unigenes/transcripts (n) & 83,493 & 103,196 \\
Number of unigenes/transcripts mapped to TAIR CDS (n) & 52,864 & 70,753 \\
Total number of CDS in TAIR (n) & 35,386 & 35,386 \\
Total number of mapped CDS in TAIR(n) & 22,435 & 22,433 \\
Total length of CDS in TAIR (bp) & $43,546,300$ & $43,546,300$ \\
Total length of mapped CDS in TAIR (bp) & $31,264,828$ & $31,145,430$ \\
Total length of unigenes/transcripts (bp) & $26,651,285$ & $71,935,591$ \\
Total length of mapped unigenes/transcripts(bp) & $18,193,518$ & $55,883,786$ \\
Total length of overlapping sequences (bp) & $15,234,784$ & $44,020,562$ \\
Percentage of mapped CDS number & $22,435 / 35,386=63.4 \%$ & $22,433 / 35,386=63.4 \%$ \\
\hline
\end{tabular}


Table 4 Summary of Camelina unigene annotations from SOAPdenovo assembly

\begin{tabular}{|c|c|c|c|}
\hline & $\begin{array}{l}\text { Unigenes } \\
\text { (n) }\end{array}$ & $\begin{array}{l}\text { Annotations } \\
\text { (n) }\end{array}$ & $\begin{array}{l}\text { Functional } \\
\text { classification }\end{array}$ \\
\hline All assembled unigenes & 83,493 & - & - \\
\hline $\begin{array}{l}\text { Gene annotations against } \\
\text { Arabidopsis thaliana protein } \\
\text { of NR }\end{array}$ & 45,307 & 45,307 & - \\
\hline $\begin{array}{l}\text { Gene annotations against } \\
\text { Arabidopsis lyrata protein } \\
\text { of NR }\end{array}$ & 16,969 & 16,969 & - \\
\hline $\begin{array}{l}\text { Gene annotation against } \\
\text { Brassica protein of NR }\end{array}$ & 722 & 722 & - \\
\hline $\begin{array}{l}\text { Unique gene annotations } \\
\text { against plant NR }\end{array}$ & 67,497 & 67,497 & - \\
\hline $\begin{array}{l}\text { Gene annotation against } \\
\text { Swiss-Prot }\end{array}$ & 40,804 & 40,804 & - \\
\hline $\begin{array}{l}\text { Gene annotation against } \\
\text { COG }\end{array}$ & 14,190 & 14,190 & 25 categories \\
\hline $\begin{array}{l}\text { Gene annotation against } \\
\text { KEGG }\end{array}$ & 27,042 & 27,042 & $\begin{array}{l}119 \\
\text { pathways }\end{array}$ \\
\hline $\begin{array}{l}\text { GO annotations for } \\
\text { biological process }\end{array}$ & 23,524 & 49,164 & $\begin{array}{l}27 \text { sub- } \\
\text { categories }\end{array}$ \\
\hline $\begin{array}{l}\mathrm{GO} \text { annotations for cellular } \\
\text { component }\end{array}$ & 25,885 & 37,439 & $\begin{array}{l}9 \text { sub- } \\
\text { categories }\end{array}$ \\
\hline $\begin{array}{l}\text { GO annotations for } \\
\text { molecular function }\end{array}$ & 26,825 & 30,888 & $\begin{array}{l}10 \text { sub- } \\
\text { categories }\end{array}$ \\
\hline All annotated unigenes & 67,791 & - & - \\
\hline $\begin{array}{l}\text { Unigenes matching all four } \\
\text { databases }\end{array}$ & 11,685 & - & - \\
\hline
\end{tabular}

1.0E-50-1.0E-5 (Figure 1a). The translated amino acid sequences of unigenes were also closely similar to sequences from the NR database; nearly $90 \%$ of the BLASTX hits were in a similarity range between $40 \%$ and $100 \%$. Only $9.6 \%$ of hits had similarity values less than 40\% (Figure 1c). Homologies between different species are depicted in Figure 1e. Among hits, 67.1\% matched to Arabidopsis thaliana, followed in sequence by A. lyrata (25.1\%) and Brassica (1.1\%) (Figure 1e).

We also matched protein coding sequences of unigenes with the protein database at Swiss-Prot using BLASTX; 40,804 of 83,493 unigenes (48.87\%) returned hits at an E-value threshold of $\leq 1.0 \mathrm{E}-5$ (Table 4). Most of the matched sequences (79.2\%) had E-values between $1.0 \mathrm{E}-50$ to $1.0 \mathrm{E}-5$, and the remaining $20.8 \%$ had strong homologies with E-values of $\leq 1.0 \mathrm{E}-50$ (Figure 1b). The similarity frequency distribution against Swiss-Prot was different from that obtained against the NR database; $86.9 \%$ of query sequences against Swiss-Prot had similarities between $20 \%$ and $80 \%$; only $13.1 \%$ of sequences had strong homologies with $>80 \%$ identity (Figure 1d).

\section{Conserved domain annotation and COG classification}

COG is a database built on phylogenetic relationships of protein sequences from 66 genomes, including bacteria, plants and animals. Individual proteins or paralogs from at least three lineages are categorized in each COG to represent an ancient conserved domain. Within the Camelina sativa unigene set, 14,190 were categorized (E-value $\leq 1.0 \mathrm{E}-5)$ in 25 functional COG clusters (Table 4, Figure 2). Thus, only a small proportion of the unigenes (17.0\%) carried protein domains with annotation for COG categories. The five largest categories were: 1) general function predictions only (15.6\%), 2) post-translational modification, protein turnover, chaperones $(8.6 \%), 3)$ transcription $(8.3 \%), 4)$ translation, ribosomal structure and biogenesis (7.7\%), and 5) replication, recombination and repair (7.5\%).

\section{Gene ontology (GO) classification}

To functionally categorize Camelina sativa unigenes, we assigned GO terms to each assembled unigene [14]. The Camelina unigenes were categorized in three main GO categories: biological process $(23,524,30.9 \%)$, cellular component $(25,885,34.0 \%)$ and molecular function $(26,825,35.2 \%)$. These GO terms were further subdivided into 46 sub-categories (Table 4 and Figure 3). In total, 33,475 unigenes were assigned to at least one of the GO categories of biological process, cellular component and molecular function (Additional file 1: Figure S2). The transcriptome of Camelina sativa was closely related to Arabidopsis thaliana sequences (Figure 1e). Only $1.1 \%$ of unigenes (722 unigenes) had higher homology with genes from Brassica spp. A substantial number (190) of these 722 unigenes were annotated as disease resistance proteins. They accounted for $26.3 \%$ of total unigenes annotated to Brassica sequences (Figure 4 and Additional file 3). After overlapping unigenes were filtered, 381 individual NR IDs matched Brassica sequences; among these, 50 NR IDs matched Brassica disease resistance proteins (Additional file 3).

\section{KEGG pathway mapping}

Unigenes of Camelina sativa were mapped to KEGG pathways by using the translated amino acids to run BLASTX against the KEGG database. All returned hits with an E-value $\leq 1.0 \mathrm{E}-5$ were annotated with Enzyme Commission (EC) numbers [15]. Using Arabidopsis thaliana as a reference for pathways analysis, we annotated and mapped 27,042 of 83,493 unigenes (32.4\%) to EC numbers in 119 KEGG pathways (Figure S3 in Additional file 1 and Additional file 4). Metabolic pathways had the largest number of unigenes $(6,469$ members, 23.9\%, ko01100), followed in sequence by secondary metabolite biosynthesis (3,600 members, $13.3 \%$, ko01110), plant-pathogen interaction $(2,004$ 

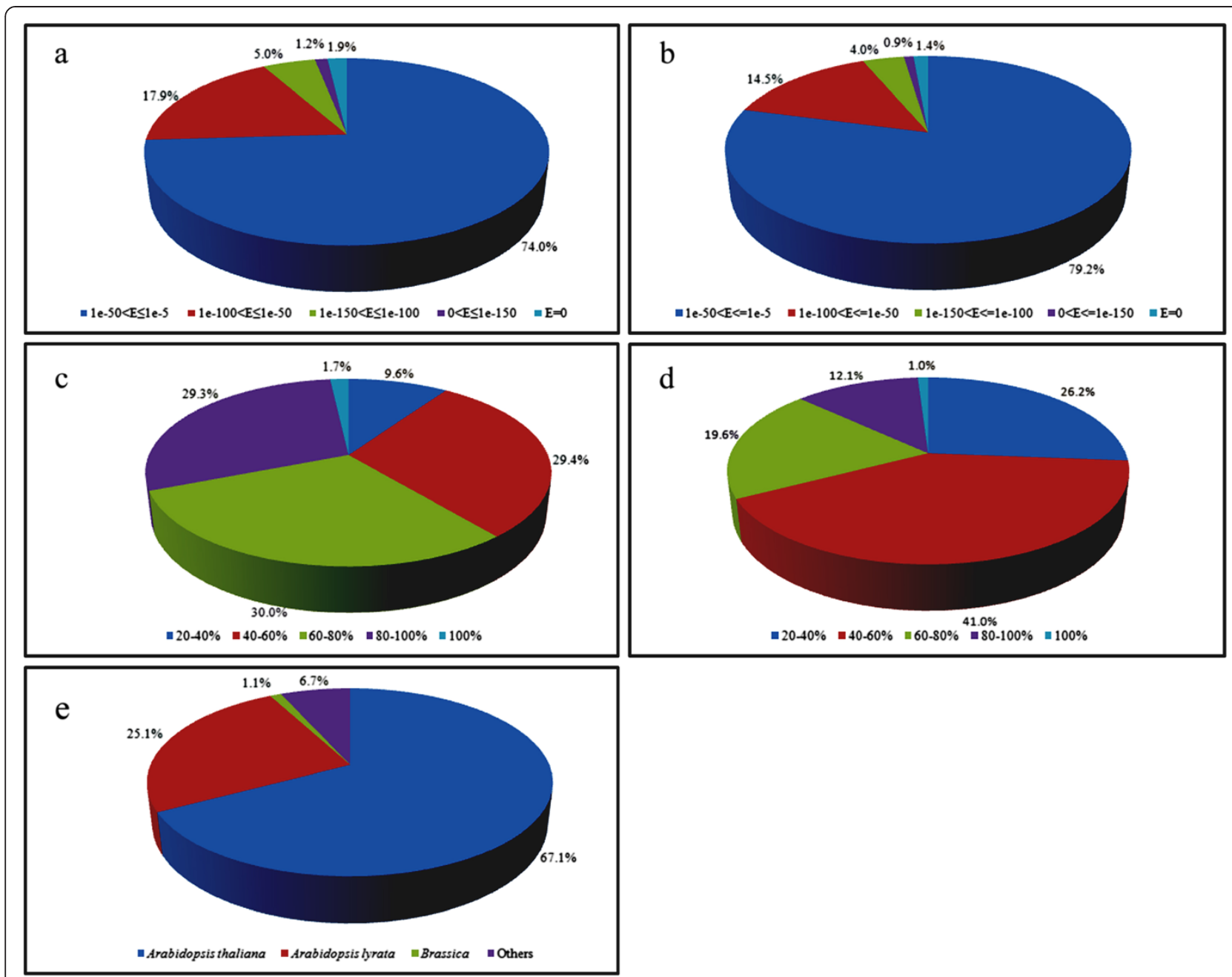

Figure 1 Unigene homology searches against NR and Swiss-Prot databases. (a) E-value proportional frequency distribution of BLAST hits against the NR database. (b) E-value proportional frequency distribution of BLAST hits against the Swiss-Prot database. (c) Proportional frequency distribution of unigene similarities against the NR database based on the best BLAST hits (E-value $\leq 1.0 \mathrm{E}-5$ ). (d) Proportional frequency distribution of unigene similarities against the Swiss-Prot database based on the best BLAST hits (E-value $\leq 1.0$ E-5). (e) Proportional homology distribution among other plant species based on the best BLAST hits against the NR database (E-value $\leq 1.0 \mathrm{E}-5$ ).

members, 7.4\%, ko04626), spliceosome (1,333 members, $4.9 \%$, ko03040), protein processing in the endoplasmic reticulum (973 members, 3.6\%, ko04141), and starch and sucrose metabolism (777 members, 2.9\%, ko00500) (Additional file 4).

Overall, 67,791 unique sequence-based annotations had BLASTX scores with E-values $\leq 1.0 \mathrm{E}-5$ in the NR, Swiss-Prot and KEGG databases (Additional file 1: Figure S3). Among this number, 11,685 unigenes had hits in all four public databases (NR, COG, Swiss-Prot and KEGG), with relatively well defined functional annotations (Table 4, Figure S3 in Additional file 1). These annotations provide a valuable resource for investigating specific processes, structures, functions, and pathways that will guide research on Camelina sativa.

\section{Protein coding sequence (CDS) prediction}

Unigenes were firstly aligned by BLASTX (E-value $\leq$ $1.0 \mathrm{E}-5)$ to protein databases in the priority order NR, Swiss-Prot, KEGG, COG. Unigenes aligned to a high priority database were not aligned to databases of lower priority. The process ended when all alignments had been performed. The correct reading frame of the nucleotide sequences of unigenes (5'-3' direction) was defined by the highest rank in the BLAST results, and the corresponding protein sequences were obtained from the standard codon table. Unigenes that could not be aligned to any database were scanned with ESTScan [16] to produce the nucleotide and amino acid sequences of the predicted region. In total, CDS of 67,739 unigenes were generated by BLASTX protein database 


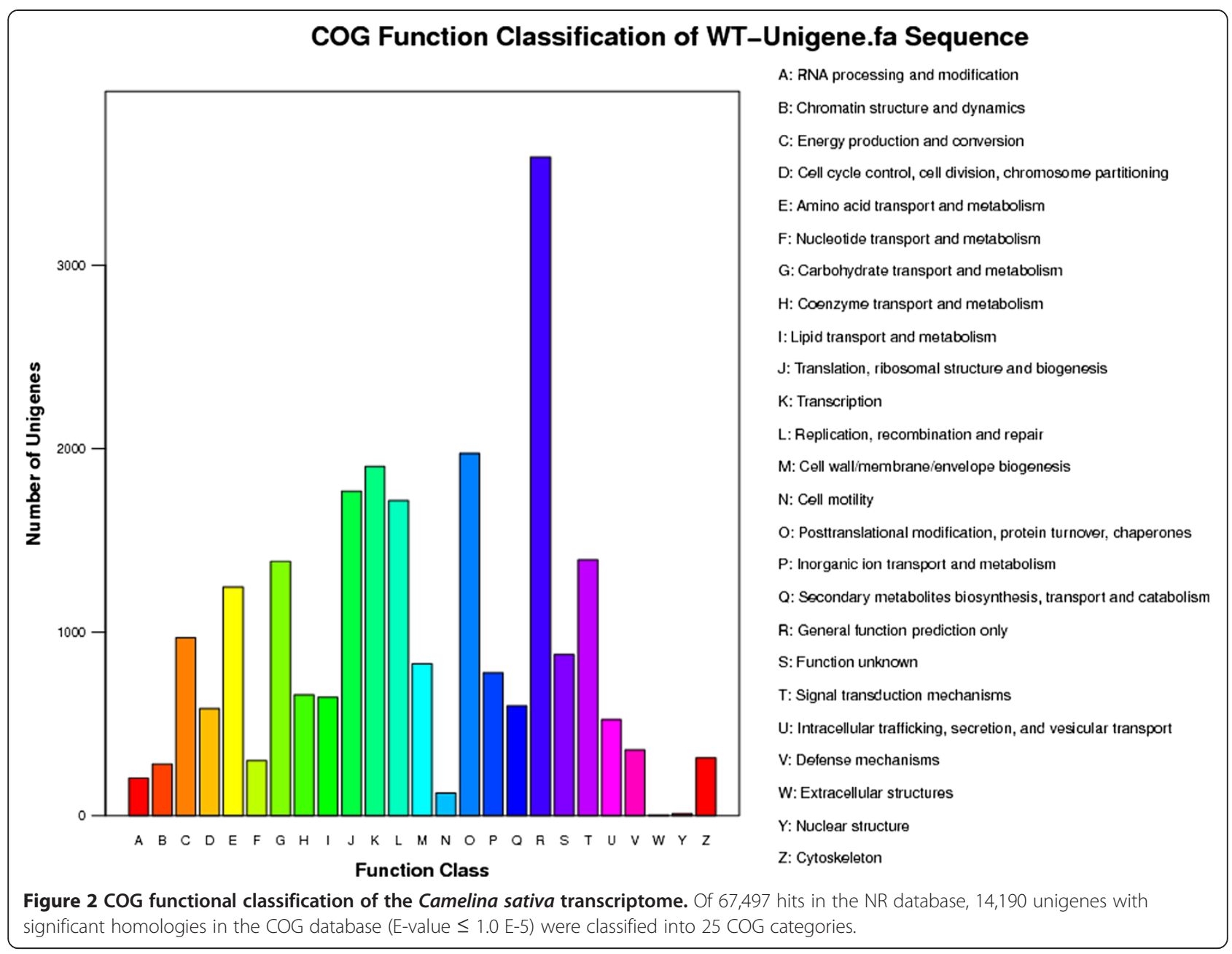

searches described above. To evaluate the quality of the databases, we analyzed the ratio of gap lengths to the sizes of unigene CDS. Almost all of the unigenes had gap lengths that were $<5 \%$ of total length, accounting for $99.96 \%$ of total unigenes with CDS sequences (67,739 unigenes) (Additional file 1: Figure S4a). The majority of the unigene CDS (88.30\%) had < 500 bp. Of 67,739 unigenes with CDS sequences, 7,923 had $\geq 500 \mathrm{bp}$ and 1,340 had $\geq 1000 \mathrm{bp}$. The size frequency distributions of these unigene CDS and proteins are depicted in Figure S4b and S4c, respectively (Additional file 1). We scanned 1,042 CDS of unigenes that could not be aligned to any database by ESTScan. Of these, only two had gaps (Additional file 1: Figure S4d). The majority of the unigene CDS assigned by ESTScan (96.93\%) were shorter than 500 bp (Additional file 1: Figure S4e); this was also the case for protein sequences obtained from ESTScan (Additional file 1: Figure S4f).

\section{Fatty acid pathways in Camelina sativa and Arabidopsis} thaliana

Fatty acids are carboxylic acids with long hydrocarbon chains that are saturated or unsaturated. The carbon atoms per molecule vary from 10 to 30 . In Camelina seeds, most of the fatty acids are unsaturated and the oil has high omega-3 fatty acid content (35-40\%). Eggs from hens and milk from cows fed Camelina meal contain elevated contents of unsaturated fatty acids $[17,18]$. In our annotated Camelina sativa transcriptome unigene dataset, we identified key genes involved in fatty acid biosynthesis and unsaturated fatty acid biosynthesis (Additional file 5). Camelina sativa shares pathways similar to those of Arabidopsis thaliana [19]. However, not all gene members in the Arabidopsis genome were identified in the transcriptome dataset. For example, there are 7 homologous stearoyl-ACP desaturase genes (FAB2, DES1-6) in the genome of Arabidopsis [20], but only four of these can find highly homologous unigenes (E-values $\leq 1.0 \mathrm{E}-68$ ) in the transcriptome dataset (data not shown). The other three members may not be expressed in leaves, or may be absent from the genome of $C$. sativa.

\section{Discussion}

High-throughput sequencing is a superior technology for transcriptome analysis. Compared with traditional 


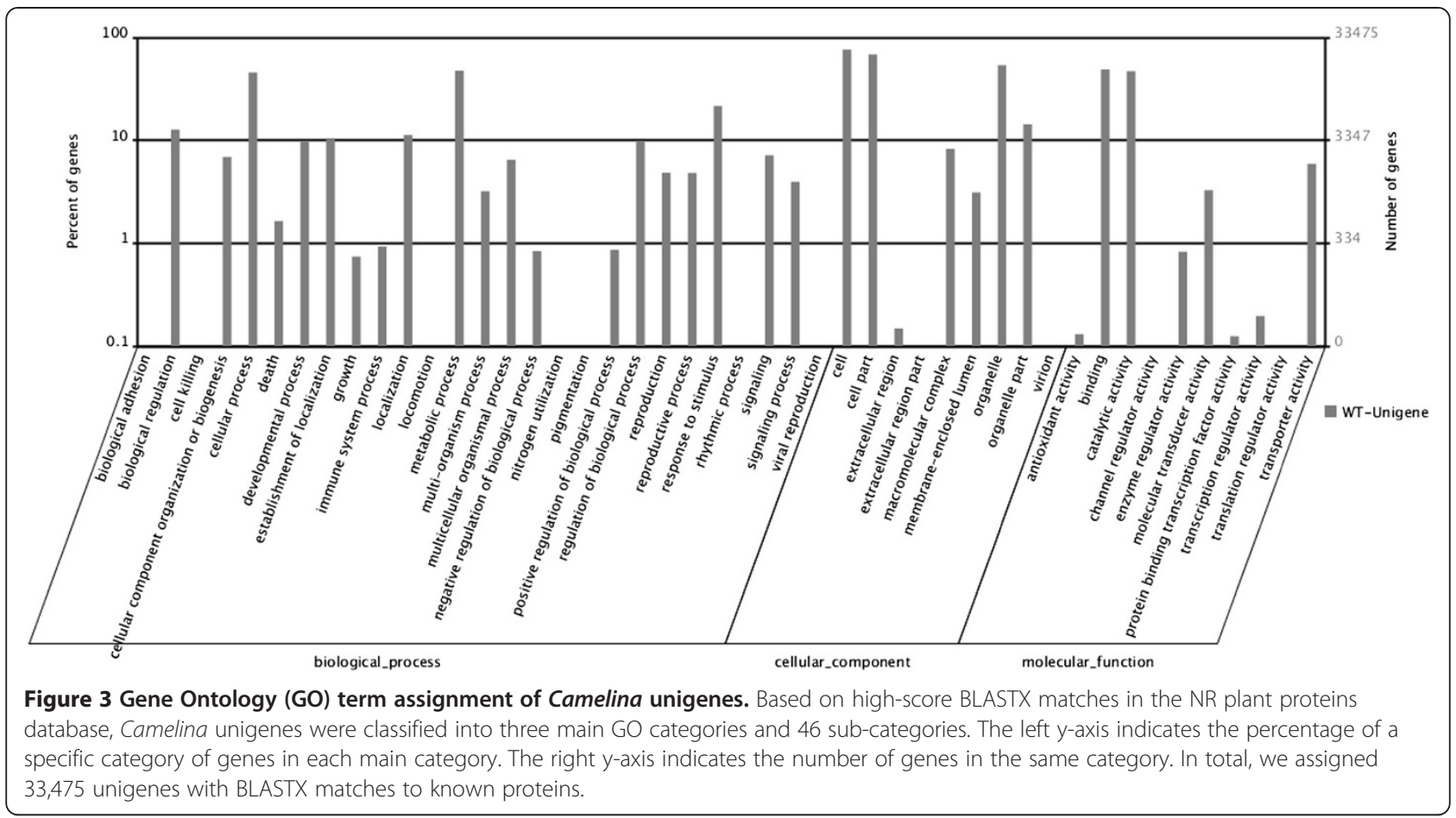

large-scale EST sequencing, it is less costly and more efficient. It is more suitable for use in non-model organisms whose genomic sequences are unknown. Prior to our work, there was no sequencing information on Camelina sativa in public databases. We used RNA-seq technology for transcriptome profiling to sequence RNA extracted from leaves. This de novo transcriptome sequencing technology has been used for many plants, including rice [21], maize [22], sesame [23], bamboo
[24], poplar [25]. However, Illumina transcriptome analysis has been restricted previously to the sequencing of organisms for which reference genomes were available [26]. The Illumina sequence technology has improved recently, most notably through an expanded capability for de novo sequencing of relatively short reads and their assembly into unigenes by paired-end sequencing technology [27]. Paired-end sequencing not only increases the depth of sequencing, but also improves de

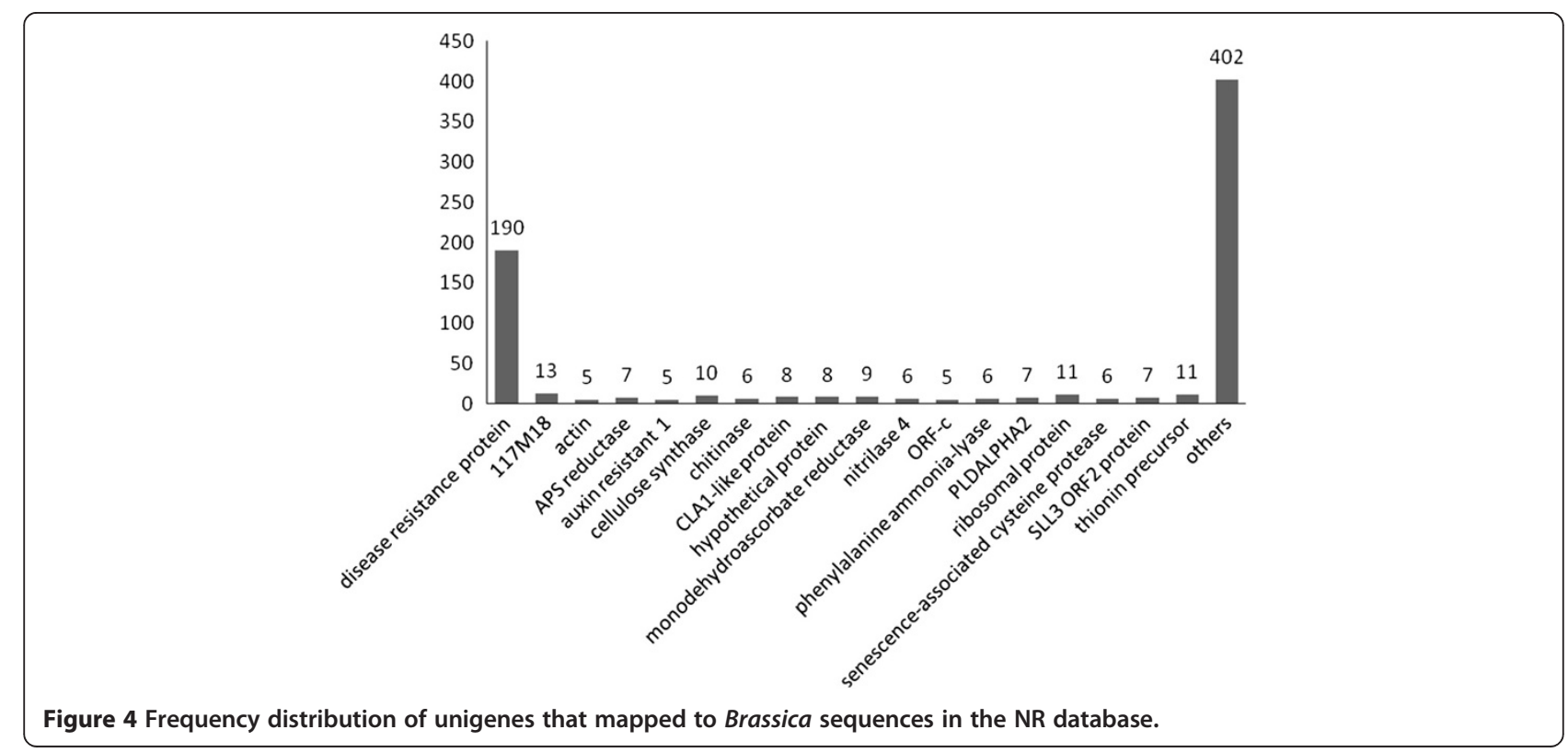


novo assembly efficiency. Through this procedure, we obtained $2.42 \mathrm{G}$ bp reads with 27.0 million clean reads. All of these reads were assembled into 83,493 unigenes by SOAPdenovo and 103,196 transcripts by Trinity. While the output was consistent with a previous study on the Camellia sinensis transcriptome [28], the hexaploid genome of Camelina sativa add complexity to the analysis [29]. When the assembled 83,493 unigenes and 103,196 transcript were mapped to Arabidopsis CDS (TAIR 10), 22,435 and 22,433 unique Arabidopsis CDS could be mapped, respectively (Table 3). Multiple unigenes could be mapped to the same CDS as they cover different regions of the CDS; and 2 or 3 unigenes could be mapped to the same CDS due to the polyploidy of Camelina. Six Camelina genes expressed in developing seeds were shown to have 3 haplotypes in ecotype CS32 [29]. To confirm that Camelina ecotype MT-5 is also a hexaploid, these 18 sequences were mapped to the clean reads and the unigenes/transcripts produced by SOAPdenovo and Trinity by BLASTN with perfect match and E-value $\leq 1.0 \mathrm{E}-5$. Three out of the six genes could find 3 haplotypes from our clean reads database, confirming that ecotype MT-5 is also a hexaploid (data not shown). Some unigenes/transcripts are actually different haplotypes of the same gene. For example, three unigenes (Unigene5348_WT, Unigene30695_WT, Unigene34277_WT) were found to be the three haplotypes of the homologous gene of AT2G18040. This reflects the complexity of assembling polyploidy transcriptome. Currently no existing assembler can handle polyploidy well.

Transcriptome analysis indicates that Camelina sativa is closely related to Arabidopsis thaliana (Figure 1e). A particular example of this relatedness is the presence of AtPAP2(AT1G13900)-like (CsPAP2, unigenes 87, 6572, 7242, 25505, 46194, 52973 and 76943) and AtPAP9 (AT2G03450)-like (CsPAP9, unigenes 129, 27542, 29608, 33278, 39915, 40207, 58111, 63089, 63449, 73151) sequences in the transcriptome of Camelina sativa (data not shown). CsPAP2 shares 91\% amino acid sequence identity with AtPAP2; CsPAP9 shares 89\% amino acid sequence identity with AtPAP9. In Arabidopsis and Camelina, two PAPs carry a C-terminal hydrophobic motif, but only one PAP-like sequence is known to carry this hydrophobic motif at the C-terminus in other plant genomes. For example, of 35 PAP-like genes in the soybean genome, only one PAP sequence (GmPAP35) carries this C-terminal motif [30]. Overexpression of AtPAP2 in A. thaliana produces fast-growth and high seed-yield phenotypes [31]. Overexpression of AtPAP2 in Camelina sativa also produces fast-growth and high seed-yield phenotypes [32]. It would be worthwhile investigating whether this effect is specific only to A. thaliana and C. sativa. Of particular interest would be identification of the physiological mechanism underlying this difference among plant species. Is this merely the result of a random gene duplication event during the evolution of $A$. thaliana and $C$. sativa, and what is the physiological function of the single PAP sequence with the specific C-terminal hydrophobic motif in other plant species?

Due to their close genetic relationship, Camelina sativa and Arabidopsis thaliana share similar KEGG pathways. We generated 119 KEGG pathways from the C. sativa transcriptome and compared them with pathways in A. thaliana. Some pathways in the two species were identical, including pathways involved in biosynthesis of unsaturated fatty acids, biotin metabolism, citrate cycle (TCA cycle), fatty acid elongation in mitochondria, glycosaminoglycan degradation, proteasome and protein export. Moreover, except in a few cases, most of the genes involved in these pathways were highly similar between the two species (Additional file 6).

While most of the unigenes had high sequence identity to Arabidopsis sequences (Figures 1e, f), a small proportion $(1.1 \%)$ of defense-related unigenes were closely similar to Brassica sequences (Figure 4 and Table 4). We identified a non-trivial proportion of these as disease resistance genes. Plants have developed defense systems for protection against pathogen invasion. In plant genomes, the nucleotide-binding site (NBS)-encoding resistance genes belong to one of the largest gene families and account for approximately $2 \%$ of all genes. NBS-encoding genes of some monocotyledons and dicotyledons, including Oryza sativa [33], Medicago truncatula [34], Brassica rapa [35], and Populus trichocarpa [36], have been studied using model species as reference organisms. In B. rapa, 92 non-redundant NBS-encoding genes have been identified [35]. Of 722 Camelina unigenes that matched Brassica sequences, 190 were disease resistance genes (including $\mathrm{BrCN}, \mathrm{BrCNL}, \mathrm{BrNL}, \mathrm{BrTN}, \mathrm{BrTNL}$ and LRR) (Additional file 3). The conservation of these disease-resistance genes in Camelina over evolutionary time scales implies that Camelina and B. rapa have been exposed to the threats from closely-related pathogens.

Glucosinolates are secondary metabolites of the Brassicales that play important roles in plant defense. Most genes that encode glucosinolates biosynthesis in Arabidopsis have been clearly identified and may be used as reference loci for research on Camelina glucosinolate genes $[37,38]$. We screened all unigenes of $C$. sativa by matching them to the query sequence of Arabidopsis thaliana genes using BLASTX tools. Except for AOP3, CYP79F2, MYB76 and FMO GS-OX2 4, all homologs of glucosinolate synthesis genes in A. thaliana are found in Brassica rapa [39]. However, we found homologs of AOP3, CYP79F2, MYB76 and 
$F M O_{G S-O X 2 \sim 4}$ in the transcriptome of C. sativa (data not shown), again confirming the close relationship between this species and $A$. thaliana.

\section{Conclusions}

Using the Illumina platform, we derived by paired-end and de novo technology a dataset comprising 83,493 unigenes from the Camelina sativa transcriptome. Of these, 67,791 were annotated with gene descriptions from NR, Swiss-Prot, COG and KEGG databases. After removal of redundancies, 25,329 non-redundant sequences were obtained. We discovered differences between KEGG pathways of A. thaliana and C. sativa (Additional files 4 and 6). Though both of these species belong to the Brassicaceae and share a close genetic relationship, there was inter-specific variation in genes involved in some metabolic pathways. This is a first report of Camelina sativa transcriptome analysis; it provides a valuable database for further research on this species.

\section{Methods}

RNA preparation from plant materials

Camelina sativa ecotype MT-5 was obtained from Dr. Alice Pilgeram, Montana State University, USA. We grew plants in a greenhouse under normal sunlight in Hong Kong, China. Both young and mature leaves were collected for RNA extraction. Leaves we collected were immediately frozen in liquid nitrogen and stored at $-80^{\circ} \mathrm{C}$ until further use. For Illumina sequencing, total RNA was extracted from pooled leaves and then digested with DNase I following manufacturer's instructions (RNeasy Plant Mini Kit, Qiagen, Hong Kong). RNA was quantified with an Agilent 2100 Bioanalyzer RNA Nanochip. At least $20 \mu \mathrm{g}$ of total RNA at a concentration of $\geq 400 \mathrm{ng} / \mu \mathrm{l}$, OD260/280 $=1.8 \sim 2.2$, RNA $28 \mathrm{~S}: 18 \mathrm{~S} \geq 1.0$, and RNA Integrity Number $(\mathrm{RIN}) \geq 7.0$ were used for cDNA library preparation.

\section{Preparation of cDNA library for transcriptome sequencing} Illumina sequencing was performed at the Beijing Genomic Institute, Shenzhen, China following manufacturer's instructions. First, poly-T oligo-attached magnetic beads (Illumina) were used to isolate poly $(\mathrm{A})^{+}$RNA from the total RNA quantified. Subsequently, poly $(A)^{+}$RNA was purified and fragmented into smaller pieces (200-700 nt) using divalent cations at $94^{\circ} \mathrm{C}$ for exactly 5 minutes. Firststrand cDNA was synthesized with SuperScript II reverse transcriptase and random primers using the small fragment RNAs as templates. Second-strand cDNA was then synthesized using GEX second strand buffer, dNTPs, RNase $\mathrm{H}$ and DNA polymerase I. These cDNA fragments were further processed with end repair and phosphorylation using T4 DNA polymerase, Klenow DNA polymerase, and T4 polynucleotide kinase. The repaired cDNA fragments were
3' adenylated using Klenow Exo-; Illumina's paired-end adapters were ligated to the ends of these 3'-adenylated cDNA fragments. The products from this ligation reaction were electrophoresed on a $2 \%(\mathrm{w} / \mathrm{v})$ TAE-agarose gel and purified to select templates of different sizes for downstream enrichments. cDNA fragments of $200 \mathrm{bp}( \pm 25 \mathrm{bp}$ ) were excised from the gel. To enrich the quantity of the cDNA template, fifteen cycles of PCR amplification were performed using PCR primers PE1.0 and PE2.0 with Phusion DNA Polymerase. Finally, a cDNA library was constructed with a fragment length range of $200 \mathrm{bp}$ ( $\pm 25 \mathrm{bp}$ ).

\section{Data filtering and de novo assembly by SOAPdenovo}

The raw "reads" were filtered to obtain high quality de novo transcriptome sequence data. First, all reads with adaptor contamination were discarded. Second, reads with unknown nucleotides comprising more than $5 \%$ were removed. Third, low-quality reads with ambiguous sequence "N" were discarded. Subsequently, de novo assembly of the clean reads was performed using SOAPdenovo, which first combined reads with selected overlap lengths to form longer fragments without $\mathrm{N}$; these fragments are termed contigs. The reads with overlapping regions were then mapped back to the contigs for scaffold construction; with paired-end reads it is possible to detect contigs from the same transcript and the distances between them. In the next step, SOAPdenovo connected the contigs using $\mathrm{N}$ to represent unknown sequences between each pair of contigs to produce scaffolds. Paired-end reads were used again to fill the gaps in the scaffolds and get sequences with the fewest Ns that could be extended from either end. These sequences are termed unigenes. Finally, sequences of assembled unigenes were translated into amino acids in order to run BLASTX at an E-value threshold of 1.0E-5 following priorities of the non-redundant protein (NR) database (http://www.ncbi.nlm.nih.gov), the Swiss-Prot protein database (http://www.expasy.ch/sprot), the Kyoto Encyclopedia of Genes and Genomes (KEGG) pathway database (http://www.genome.jp/kegg) and the Cluster of Orthologous Groups (COG) database (http://www.ncbi. nlm.nih.gov/COG). The best aligning result was used to decide the sequence direction of each unigene. If a unigene had no alignment hit (match) in the above databases, ESTScan software [16] was used to predict the CDS domain and its direction. For unigenes with sequence direction, sequences from the 5' end to the $3^{\prime}$ end were provided. For those without sequence direction, we obtained sequences from assembly software. TIGR Gene Indices Clustering Tools (TGICL) software [40] was used to clusters scaffolds to produce unigenes with the fewest redundant sequences. 


\section{de novo assembly by Trinity}

In order to verify transcriptome assembly by SOAPdenovo, we ran Trinity [13] to assemble clean reads into transcripts. Trinity focuses more on splice isoforms. It first forms contigs that represent the significant parts of individual isoforms. Then, it clusters the contigs into individual groups such that contigs from isoforms likely to be from the same gene are grouped together. Lastly, each group of contigs is processed separately. Furthermore, Trinity is based on paired-end reads and attempts to reconstruct transcripts for splice isoforms in each group. Different from SOAPdenovo, Trinity did not fill gaps with 'N's to represent unknown sequences when two contigs were connected. Instead, Trinity built a K-mer dictionary from all clean reads based on frequency and considered each K-mer as an initial contig. Each initial contig (in descending frequency order) was extended by selecting the most frequent K-mer in the dictionary with $\mathrm{K}-1$ overlaps with the current contig end, until neither direction could be extended further. Subsequently, contigs were pooled if they shared at least one K-1-mer and there were reads across the junction sites. A de Brujin graph was constructed for each contig pool. Finally, each de Brujin graph was compacted and linear sequences representing each alternative splicing form and/or high similar transcripts were produced.

\section{Unigene annotation and classification}

The annotation of unigenes was performed using various bioinformatics procedures. Unigenes were firstly translated into amino acids in six frames and aligned with BLASTX to protein databases including NR, Swiss-Prot, KEGG and COG (E-value $\leq 1.0 \mathrm{E}-5)$; the protein with highest sequence similarity was retrieved and annotated to each unigene. With NR annotation, Blast2GO [41] software was used to get $\mathrm{GO}$ annotation defined by molecular function, cellular component and biological process ontologies. After obtaining GO annotation for every unigene, WEGO software [42] was used to produce GO functional classification for all unigenes and to interpret the distribution of species' gene functions at the macro level. Unigene sequences were also aligned to the COG database to predict and classify possible functions. Pathway assignments were determined following the Kyoto Encyclopedia of Genes and Genomes pathway database using BLASTX with an E-value threshold of 1.0E-5.

\section{Additional files}

Additional file 1: Supplementary Figures for this study. Figure S1

Overview of assembly by SOAPdenovo. (a) Length frequency distribution of contigs obtained from de novo assembly of high-quality clean "reads". (b) Length frequency distribution of gap ratios ( $\mathrm{N} / \mathrm{size}$ ) in assembled scaffolds. (c) Frequency distribution of assembled scaffold lengths. (d)
Length frequency distribution of unigenes produced by contig joining, gap filling, and scaffold clustering. (e) Gap frequency distribution of assembled unigenes. $x$-axis values are ratios of gap length to length of assembled unigenes. $y$-axis values are frequencies of unigenes containing gaps. (f) Random frequency distribution of Illumina sequencing reads in assembled unigenes. $x$-axis values are relative positions of sequencing reads in assembled unigenes. The orientation of unigenes is from the $5^{\prime}$ end to the $3^{\prime}$ end. Figure S2 Venn Diagrams of the three categories of GO. In total, 33,475 unigenes were assigned to at least one GO category. Figure S3 Venn diagram results from diverse databases. (a) Venn diagram showing the number of unigenes matched to sequences in NR, Swiss-Prot and KEGG databases. All annotations were based on best BLASTX hits with E-Values $\leq 1.0 \mathrm{E}-5$. The overlapping regions represent the number of unigenes that matched in different databases. (b) Venn diagram showing the number of unigenes in NR, Swiss-Prot, KEGG and COG databases. All annotations were based on the best BLASTX hits with E-Values $\leq 1.0 \mathrm{E}-5$. Figure S4 Camelina sativa transcriptome coding sequence (CDS) predicted by BLASTX and ESTScan software. (a) Number of predicted CDS with gap ratio frequency distribution (N/size). (b) Length frequency distribution of predicted CDS. (c) Length frequency distribution of predicted protein sequences. (d) Gap ratio frequency distribution of CDS predicted by ESTScan software. (e) Length frequency distribution of CDS predicted by ESTScan software. (f) Length frequency distribution of protein sequences predicted by ESTScan software.

Additional file 2: NR annotations of Camelina sativa with an E-value threshold of $1.0 \mathrm{E}-5$.

Additional file 3: 722 unigenes annotated to Brassica and 190 annotated to Brassica disease resistance protein coding genes. Additional file 4: 119 KEGG pathways with pathway ID and KO information.

Additional file 5: Number of annotated unigenes involved in fatty acid synthesis in Camelina sativa.

Additional file 6: Differences in 119 KEGG pathways between Camelina sativa and Arabidopsis thaliana.

Competing interests

The authors declare that they have no competing interests.

\section{Authors' contributions}

BLL: designed the experimental plan. CL: carried out the experiments, analyzed the data and wrote the manuscript. XL: carried out Trinity assembly analysis. SMY: verified SOAPdenovo assembly results by Trinity and revised the manuscript. All authors read and approved the final manuscript.

\section{Acknowledgements}

We thank the Beijing Genomic Institute in Shenzhen, China for sequencing and the related bioinformatics analysis. This research was supported by the General Research Fund (HKU772710M) and the Initiatives for Clean Energy and Environment (ICEE) of the University of Hong Kong.

\section{Author details}

${ }^{1}$ School of Biological Sciences, the University of Hong Kong, Pokfulam, Hong Kong, China. ${ }^{2}$ Department of Computer Science, the University of Hong Kong, Pokfulam, Hong Kong, China.

Received: 26 March 2012 Accepted: 14 January 2013

Published: 5 March 2013

\section{References}

1. Zubr J: Oil-seed crop: Camelina sativa. Ind Crop Prod 1997, 6:113-119.

2. Agegnehu M, Honermeier B: Effects of seeding rates and nitrogen fertilization on seed yield, seed quality and yield components of false flax (Camelina sativa Crtz). Bodenkultur 1997, 48:15-21.

3. Lu C, Kang J: Generation of transgenic plants of a potential oilseed crop Camelina sativa by Agrobacterium-mediated transformation. Plant Cell Rep 2008, 27:273-278.

4. Gebauer SK, Psota TL, Harris WS, Kris-Etherton PM: n-3 fatty acid dietary recommendations and food sources to achieve essentiality and cardiovascular benefits. Am J Clin Nutr 2006, 83:1526s-1535s. 
5. Frohlich A, Rice B: Evaluation of Camelina sativa oil as a feedstock for biodiesel production. Ind Crop Prod 2005, 21:25-31.

6. Bernardo A, Howard-Hildige R, O'Connell A, Nichol R, Ryan J, Rice B, Roche E, Leahy JJ: Camelina oil as a fuel for diesel transport engines. Ind Crop Prod 2003, 17:191-197

7. Moser BR, Vaughn SF: Evaluation of alkyl esters from Camelina sativa oil as biodiesel and as blend components in ultra low-sulfur diesel fuel. Bioresour Technol 2010, 101:646-653.

8. Shonnard DR, Williams L, Kalnes TN: Camelina-Derived Jet Fuel and Diesel: Sustainable Advanced Biofuels. Environmental Progress \& Sustainable Energy 2010, 29:382-392.

9. Budin JT, Breene WM, Putnam DH: Some compositional properties of Camelina (Camelina-Sativa L Crantz) seeds and oils. J Am Oil Chem Soc 1995, 72:309-315.

10. Eden E: Variation in resistance of camelina (Camelina sativa [L.] crtz.) to downy mildew (Peronospora camelinae Gaum.). Journal of Phytopathology-Phytopathologische Zeitschrift 2001, 149:129-133.

11. Parchman TL, Geist KS, Grahnen JA, Benkman CW, Buerkle CA: Transcriptome sequencing in an ecologically important tree species: assembly, annotation, and marker discovery. BMC Genomics 2010, 11:180.

12. Sun C, Li Y, Wu Q, Luo HM, Sun YZ, Song JY, Lui EMK, Chen SL: De novo sequencing and analysis of the American ginseng root transcriptome using a GS FLX Titanium platform to discover putative genes involved in ginsenoside biosynthesis. BMC Genomics 2010, 11:262.

13. Grabherr MG, Haas BJ, Yassour M, Levin JZ, Thompson DA, Amit I, Adiconis $X$, Fan L, Raychowdhury R, Zeng QD, et al: Full-length transcriptome assembly from RNA-Seq data without a reference genome. Nat Biotechnol 2011, 29:644-652.

14. Ashburner M, Ball CA, Blake JA, Botstein D, Butler H, Cherry JM, Davis AP, Dolinski K, Dwight SS, Eppig JT, et al: Gene ontology: tool for the unification of biology. The Gene Ontology Consortium. Nat Genet 2000, 25:25-29.

15. Kanehisa M, Goto S, Kawashima S, Okuno Y, Hattori M: The KEGG resource for deciphering the genome. Nucleic Acids Res 2004, 32:D277-D280.

16. Iseli C, Jongeneel CV, Bucher P: ESTScan: a program for detecting, evaluating, and reconstructing potential coding regions in EST sequences. Proc Int Conf Intell Syst Mol Biol 1999, 7:138-148.

17. Cherian G, Campbell A, Parker T: Egg quality and lipid composition of eggs from hens fed Camelina sativa. J Appl Poultry Res 2009, 18:143-150.

18. Hurtaud C, Peyraud JL: Effects of feeding camelina (seeds or meal) on milk fatty acid composition and butter spreadability. J Dairy Sci 2007, 90:5134-5145.

19. Sharma A, Chauhan RS: In silico identification and comparative genomics of candidate genes involved in biosynthesis and accumulation of seed oil in plants. Comp Funct Genomics 2012, 2012:914843.

20. Kachroo A, Shanklin J, Whittle E, Lapchyk L, Hildebrand D, Kachroo P: The Arabidopsis stearoyl-acyl carrier protein-desaturase family and the contribution of leaf isoforms to oleic acid synthesis. Plant Mol Biol 2007, 63:257-271.

21. Zhang GJ, Guo GW, Hu XD, Zhang Y, Li QY, Li RQ, Zhuang RH, Lu ZK, He $Z Q$, Fang $X D$, et al: Deep RNA sequencing at single base-pair resolution reveals high complexity of the rice transcriptome. Genome Res 2010, 20:646-654.

22. Vega-Arreguin JC, Ibarra-Laclette E, Jimenez-Moraila B, Martinez O, VielleCalzada JP, Herrera-Estrella L, Herrera-Estrella A: Deep sampling of the Palomero maize transcriptome by a high throughput strategy of pyrosequencing. BMC Genomics 2009, 10:299.

23. Wei W, Qi X, Wang L, Zhang Y, Hua W, Li D, Lv H, Zhang X: Characterization of the sesame (Sesamum indicum L.) global transcriptome using Illumina paired-end sequencing and development of EST-SSR markers. BMC Genomics 2011, 12:451

24. Zhang YJ, Ma PF, Li DZ: High-throughput sequencing of six bamboo chloroplast genomes: phylogenetic implications for temperate woody bamboos (Poaceae: Bambusoideae). PloS One 2011, 6:e20596.

25. Qiu Q, Ma T, Hu QJ, Liu BB, Wu YX, Zhou HH, Wang Q, Wang J, Liu JQ: Genome-scale transcriptome analysis of the desert poplar. Populus euphratica. Tree Physiology 2011, 31:452-461.

26. Nagalakshmi U, Wang Z, Waern K, Shou C, Raha D, Gerstein M, Snyder M: The transcriptional landscape of the yeast genome defined by RNA sequencing. Science 2008, 320:1344-1349.
27. Maher CA, Palanisamy N, Brenner JC, Cao XH, Kalyana-Sundaram S, Luo SJ, Khrebtukova I, Barrette TR, Grasso C, Yu JD, et al: Chimeric transcript discovery by paired-end transcriptome sequencing. Proc Natl Acad Sci USA 2009, 106:12353-12358.

28. Shi CY, Yang H, Wei CL, Yu O, Zhang ZZ, Jiang CJ, Sun J, Li YY, Chen Q, Xia $T$, et al: Deep sequencing of the Camellia sinensis transcriptome revealed candidate genes for major metabolic pathways of tea-specific compounds. BMC Genomics 2011, 12:131.

29. Hutcheon C, Ditt RF, Beilstein M, Comai L, Schroeder J, Goldstein E, Shewmaker CK, Nguyen T, De Rocher J, Kiser J: Polyploid genome of Camelina sativa revealed by isolation of fatty acid synthesis genes. BMC Plant Biol 2010, 10:233.

30. Li CC, Gui SH, Yang T, Walk T, Wang XR, Liao H: Identification of soybean purple acid phosphatase genes and their expression responses to phosphorus availability and symbiosis. Ann Bot 2012, 109:275-285.

31. Sun F, Suen PK, Zhang Y, Liang C, Carrie C, Whelan J, Ward JL, Hawkins ND, Jiang L, Lim BL: A dual-targeted purple acid phosphatase in Arabidopsis thaliana moderates carbon metabolism and its overexpression leads to faster plant growth and higher seed yield. New Phytol 2012, 194:206-219.

32. Zhang Y, Yu L, Yung KF, Leung DYC, Sun F, Lim BL: Over-expression of AtPAP2 in Camelina sativa leads to faster plant growth and higher seed yield. Biotechnology for Biofuels 2012, 5:19.

33. Zhou T, Wang Y, Chen JQ, Araki H, Jing Z, Jiang K, Shen J, Tian D: Genomewide identification of NBS genes in japonica rice reveals significant expansion of divergent non-TIR NBS-LRR genes. Mol Genet Genomics 2004, 271:402-415.

34. Ameline-Torregrosa C, Wang BB, O'Bleness MS, Deshpande S, Zhu HY, Roe B, Young ND, Cannon SB: Identification and characterization of nucleotide-binding site-Leucine-rich repeat genes in the model plant Medicago truncatul. Plant Physiol 2008, 146:5-21.

35. Mun JH, Yu HJ, Park S, Park BS: Genome-wide identification of NBS-encoding resistance genes in Brassica rapa. Mol Genet Genomics 2009, 282:617-631.

36. Kohler A, Rinaldi C, Duplessis S, Baucher M, Geelen D, Duchaussoy F, Meyers BC, Boerjan W, Martin F: Genome-wide identification of NBS resistance genes in Populus trichocarpa. Plant Mol Biol 2008, 66:619-636.

37. Halkier BA, Gershenzon J: Biology and biochemistry of glucosinolates. Annu Rev Plant Biol 2006, 57:303-333.

38. Grubb CD, Abel S: Glucosinolate metabolism and its control. Trends Plant Sci 2006, 11:89-100

39. Zang YX, Kim HU, Kim JA, Lim MH, Jin M, Lee SC, Kwon SJ, Lee SI, Hong JK Park TH, et al: Genome-wide identification of glucosinolate synthesis genes in Brassica rapa. FEBS J 2009, 276:3559-3574.

40. Pertea G, Huang X, Liang F, Antonescu V, Sultana R, Karamycheva S, Lee Y, White J, Cheung F, Parvizi B, et al: TIGR Gene Indices clustering tools (TGICL): a software system for fast clustering of large EST datasets. Bioinformatics 2003, 19:651-652.

41. Conesa A, Gotz S, Garcia-Gomez JM, Terol J, Talon M, Robles M: Blast2GO: a universal tool for annotation, visualization and analysis in functional genomics research. Bioinformatics 2005, 21:3674-3676.

42. Ye J, Fang L, Zheng H, Zhang Y, Chen J, Zhang Z, Wang J, Li S, Li R, Bolund L: WEGO: a web tool for plotting GO annotations. Nucleic Acids Res 2006 34:W293-W297.

doi:10.1186/1471-2164-14-146

Cite this article as: Liang et al:: De novo assembly and characterization of Camelina sativa transcriptome by paired-end sequencing. $B M C$ Genomics 2013 14:146. 